\title{
Controllable helical deformations on printed anisotropic composite soft actuators
}

Cite as: Appl. Phys. Lett. 112, 181905 (2018); https://doi.org/10.1063/1.5025370

Submitted: 09 February 2018 • Accepted: 19 April 2018 • Published Online: 03 May 2018

Dong Wang, Ling Li, (D) Ahmad Serjouei, et al.

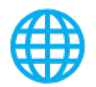

View Online

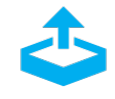

\section{ARTICLES YOU MAY BE INTERESTED IN}

Active materials by four-dimension printing

Applied Physics Letters 103, 131901 (2013); https://doi.org/10.1063/1.4819837

Shape formation of helical ribbons induced by material anisotropy

Applied Physics Letters 110, 091901 (2017); https://doi.org/10.1063/1.4977090

Bionic torus as a self-adaptive soft grasper in robots

Applied Physics Letters 116, 023701 (2020); https://doi.org/10.1063/1.5128474

\section{Challenge us.}

What are your needs for periodic signal detection?

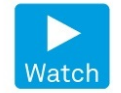

- Zurich

- Instruments

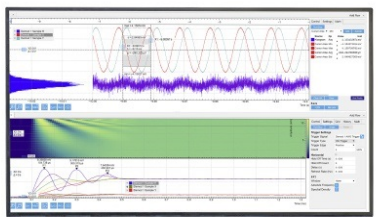

- 


\title{
Controllable helical deformations on printed anisotropic composite soft actuators
}

\author{
Dong Wang, ${ }^{1}$ Ling Li, ${ }^{2}$ Ahmad Serjouei, ${ }^{1}$ Longteng Dong, ${ }^{2}$ Oliver Weeger, ${ }^{1}$ \\ Guoying $\mathrm{Gu},{ }^{2,3, a)}$ and Qi Ge $\mathrm{e}^{1,4, a)}$ \\ ${ }^{1}$ Digital Manufacturing and Design Centre, Singapore University of Technology and Design, Singapore \\ 487372, Singapore \\ ${ }^{2}$ State Key Laboratory of Mechanical System and Vibration, School of Mechanical Engineering, \\ Shanghai Jiao Tong University, Shanghai 200240, China \\ ${ }^{3}$ Robotics Institute, School of Mechanical Engineering, Shanghai Jiao Tong University, Shanghai 200240, \\ China \\ ${ }^{4}$ Science and Math Cluster, Singapore University of Technology and Design, Singapore 487372, Singapore
}

(Received 9 February 2018; accepted 19 April 2018; published online 3 May 2018)

\begin{abstract}
Helical shapes are ubiquitous in both nature and engineering. However, the development of soft actuators and robots that mimic helical motions has been hindered primarily due to the lack of efficient modeling approaches that take into account the material anisotropy and the directional change of the external loading point. In this work, we present a theoretical framework for modeling controllable helical deformations of cable-driven, anisotropic, soft composite actuators. The framework is based on the minimum potential energy method, and its model predictions are validated by experiments, where the microarchitectures of the soft composite actuators can be precisely defined by $3 \mathrm{D}$ printing. We use the developed framework to investigate the effects of material and geometric parameters on helical deformations. The results show that material stiffness, volume fraction, layer thickness, and fiber orientation can be used to control the helical deformation of a soft actuator. In particular, we found that a critical fiber orientation angle exists at which the twist of the actuator changes the direction. Thus, this work can be of great importance for the design and fabrication of soft actuators with tailored deformation behavior. Published by AIP Publishing.

https://doi.org/10.1063/1.5025370
\end{abstract}

Helical shapes are ubiquitous in both nature and engineering where examples can be found at all length scales, including DNA strands, ${ }^{1}$ vertebrate guts, ${ }^{2,3}$ seed pods, ${ }^{4,5}$ mechanical springs, ${ }^{6}$ spiral staircases, ${ }^{7}$ and many others. ${ }^{8-11}$ In addition, helical shapes have also been exploited as efficient modes of motion and thus adopted by creatures. Examples include bacteria flagella for locomotion, ${ }^{12-14}$ plant tendrils for climbing, elephant trunks for grasping, ${ }^{15}$ and so forth. ${ }^{16-18}$

Inspired by nature, researchers and engineers have made efforts to design and fabricate soft actuators and robots that mimic those helical motions and found various applications in diverse areas such as robotics, biomedicals, and bioengineering. Examples include soft robotic grippers capable of grasping soft or fragile objects with irregular shapes, ${ }^{19,20}$ pneumatic twisting actuators for tilting micromirrors, ${ }^{21}$ active propeller blades and control surfaces on underwater vehicles exhibiting bending and twisting motions, ${ }^{22}$ and soft robotic gloves augmenting hand rehabilitation of patients with functional grasp pathologies. ${ }^{23}$ However, further advances in the design and fabrication of actuators exhibiting helical motions are hindered mainly due to the lack of efficient fabrication tools and appropriate modeling approaches. Although in the past decade several approaches were proposed to fabricate and model helical deformations, most of them were based on the idea of building internal stresses within a multilayer structure which only exhibits a one-shot

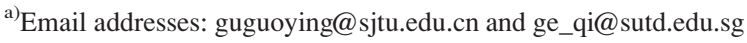

deformation to a helical shape. ${ }^{24-32}$ For example, the formations of general chiral morphologies such as nanohelices in nanomaterials induced by anisotropic surface stresses have been studied. ${ }^{32}$ Yet, these one-shot deformation approaches are not applicable for actuators and robots that require reversible and repeatable motions. In contrast, cable-driven mechanisms ensure repeatable and reversible actuation with a fast loading rate and large load capacity and are widely used in the robotics community. ${ }^{33-39}$ Nevertheless, examples of cable-driven helical motion actuators are limited as these actuators were so far mainly made of a single material, making it difficult to create anisotropy which results in helical motion. More importantly, modeling helical motions generated by cable-driven mechanisms has been elusive due to the difficulties of tracking and calculating the force exerted to the actuator from the control cable as the force direction constantly varies during helical bending/twisting.

In this letter, we present a theoretical framework based on the minimum potential energy method to model the complex helical motions of a suite of 3D printed, anisotropic, composite actuators that are controlled by a cable-driven mechanism. We start by designing the composite actuators as depicted in Fig. 1(a), where a rectangular composite $(L \times W \times H$ with $L \gg W \gg H)$ consists of an elastomeric matrix reinforced by a layer of off-centered stiff fibers. As shown in Fig. 1(b), we parameterize the composite into three layers: the top and bottom pure elastomeric layers with thicknesses $h_{1}$ and $h_{3}$, respectively, and the anisotropic middle layer with thickness $h_{2}$, which is designed through rotating a 


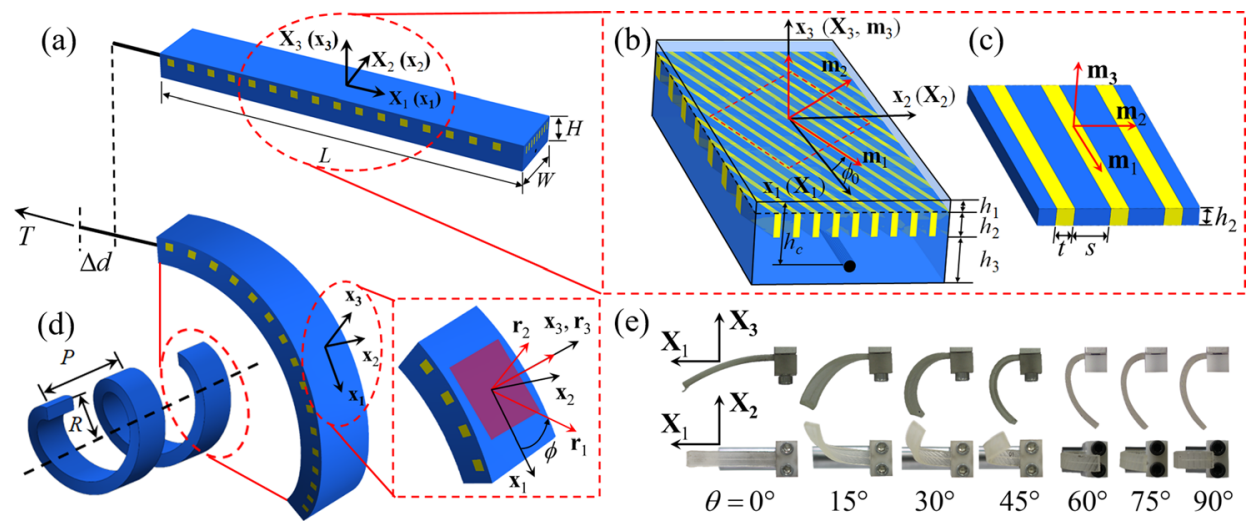

FIG. 1. (a) Schematic of a composite actuator consisting of two layers of a pure elastomer (top and bottom) and a layer of an anisotropic fiber reinforced composite (middle). (b) The detailed parameters and coordinate systems of the composite including a basic fiber reinforced composite (c) which is oriented by an angle $\phi_{0}$ with respect to the $\mathbf{x}_{1}(\mathbf{X})_{1}$-axis. (d) A helically deformed composite actuator which can be characterized by two basic parameters, pitch $P$ and radius $R$. The inset of (d) illustrates a principle strain plane $\mathbf{r}_{1}-\mathbf{r}_{2}$, where the $\mathbf{r}_{3}$-axis coincides with the $\mathbf{x}_{3}$-axis and the $\mathbf{r}_{1}$-axis rotates counter-clockwise from the $\mathbf{x}_{1}$ axis by $\phi$. (e) Experimental snapshots (front and top views) of the deformed composite actuators with a fiber orientation angle varying from $0^{\circ}$ to $90^{\circ}$ under a $10 \mathrm{~mm}$ control cable displacement.

basic fiber-reinforced composite [Fig. 1(c)] by an orientation angle $\phi_{0}$. In addition, a tiny channel with a distance $h_{\mathrm{c}}$ away from the top edge is designed all through the length direction for embedding a control cable. We fabricated the composite actuators using a commercial Polyjet multimaterial 3D printer (Stratasys, Objet500 Connex 3). During printing, we embedded the control cable into the tiny channel by interrupting the printing process. ${ }^{40}$ Based on previous studies, ${ }^{31,41}$ the layer-layer interfaces within the composites are fully bonded due to the strong covalent bonds formed during photopolymerization. There is no interaction between the control cable and the inner wall of the channel in the composite, but the cable and the composite are fully bonded at one end of the composite by superglue. Further details on the geometric parameters and fabrication process are described in the supplementary material (S1).

In Fig. 1(d), when subject to a tensile force $T$, the displacement $\Delta d$ of the control cable's free end results in a helical bending deformation of the composite actuator. This helical deformation is highly dependent on the fiber orientation angle in the middle layer $\phi_{0}$. Fig. 1(e) presents the snapshots of different deformation modes as $\phi_{0}$ varying from $0^{\circ}$ to $90^{\circ}$, and the cable displacement $\Delta d$ is kept constant at $10 \mathrm{~mm}$. We recorded the complex helical bending deformations using a self-built experimental platform (see supplementary material, S2 and S3). As shown in Fig. S2, two cameras were used to record the real-time deformation in front and top views, while a servo motor was pulling the control cable to deform the composite actuators. We acquired the deformation curves by projecting the centerline of the deformed actuator to front and top planes, respectively (Fig. S3). The two basic parameters characterizing a helical deformation, pitch $P$ and radius $R$ [Fig. 1(d)], can be calculated based on a parametric function described in supplementary material (S3).

We investigate the effects of the orientation angle $\phi_{0}$, as well as the displacement of the control fiber $\Delta d$, on the slope $S(S=P / 2 \pi R)$ and curvature $\kappa(\kappa=1 / R)$ of the helical deformation, as shown in Fig. 2. In Fig. 2(a), the slope $S$ varies with the fiber orientation angle $\phi_{0}$. At $\phi_{0}=0^{\circ}$ or $90^{\circ}$, the slope $S$ is zero, indicating that the actuators exhibit only in-plane bending. At $\phi_{0}=15^{\circ}$, the actuator deforms helically with the overall steepest, upward-directed slope [toward the positive $\mathbf{X}_{2}$ direction in Fig. 1(e)]. The upward slope decreases gradually with the increase in $\phi_{0}$ and reaches nearly zero when $\phi_{0}$ increases to $60^{\circ}$. At $\phi_{0}=75^{\circ}$, the actuator deforms helically with a downward slope [toward the negative $\mathbf{X}_{2}$ direction in Fig. 1(e)]. The curvature $\kappa$ starts at $\sim 10 \mathrm{~m}^{-1}$ when $\phi_{0}=0^{\circ}$ and reaches the maximum of $\sim 90 \mathrm{~m}^{-1}$ at $\phi_{0}=15^{\circ}$. From $\phi_{0}=30^{\circ}$ to $90^{\circ}$, the curvature $\kappa$ varies slightly around $60 \mathrm{~m}^{-1}$ to $70 \mathrm{~m}^{-1}$. In Fig. 2(b), the helical motion for increasing cable displacement $\Delta d$ from 0 to $10 \mathrm{~mm}$ is analyzed. Actuations with different fiber
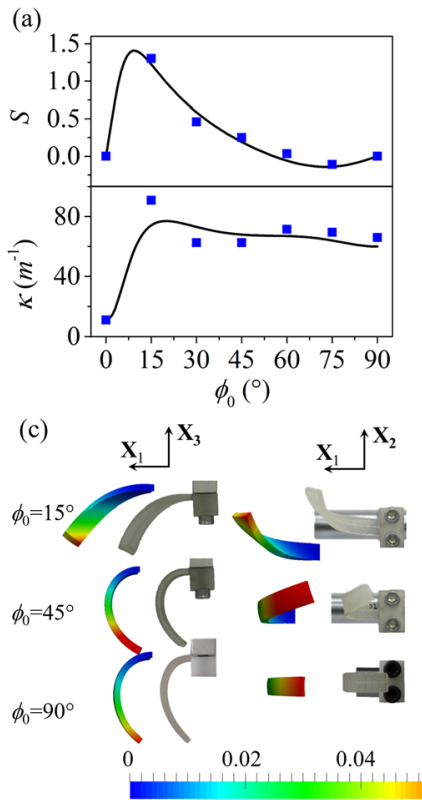

(b)

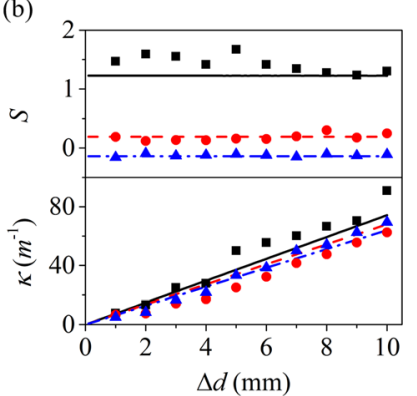

(d)

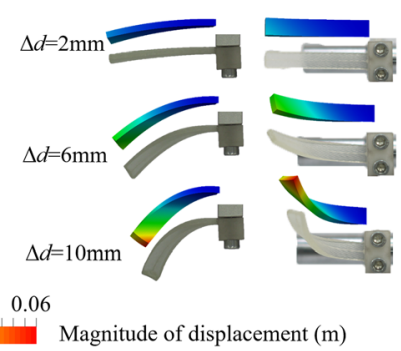

FIG. 2. (a) Effect of the fiber orientation angle $\phi_{0}$ on the slope $S$ and curvature $\kappa$ of helical motion (experimental results: blue squares and model predictions: black solid curves). (b) Effect of the control cable displacement $\Delta d$ on the slope $S$ and curvature $\kappa$ of a helical motion [experimental results: black squares $\left(15^{\circ}\right)$, red circles $\left(45^{\circ}\right)$, and blue triangles $\left(75^{\circ}\right)$; and model predictions: black solid curves $\left(15^{\circ}\right)$, red dash curves $\left(45^{\circ}\right)$, and blue dashdot curves $\left(75^{\circ}\right)$ ]. (c) and (d) Visual comparisons between experiments and 3D model simulations on the effects of $\phi_{0}$ and $\Delta d$. 
orientation angles $\phi_{0}$ possess different slopes, while the slopes stay roughly constant during the increase in the cable displacement. This indicates that the slope is an intrinsic property of a composite actuator, which is only dependent on the actuator's geometric and material properties. The curvature $\kappa$ increases linearly with the increase in the cable displacement and varies only slightly with orientation angle $\phi_{0}$.

We develop a theoretical framework based on the minimum potential energy method ${ }^{42,43}$ to model the complex helical bending deformations. During bending, the total potential energy $\Pi$ in the system can be written as

$$
\Pi=\sum_{i=1}^{3} \underbrace{\int \frac{1}{2} \boldsymbol{\varepsilon}^{i}: \mathbb{C}^{i}: \boldsymbol{\varepsilon}^{i} d V_{i}}_{U_{i}}+\underbrace{\frac{1}{2} k_{c}\left(\Delta L_{c}\right)^{2}}_{U_{c}}-\underbrace{\mathbf{F}_{c} \cdot \Delta_{c}}_{W_{c}} .
$$

$U_{i}$ is the elastic energy stored in the top $(i=1)$, middle $(i=2)$, and bottom layers $(i=3)$, which can be calculated using the corresponding strain tensor $\boldsymbol{\varepsilon}^{i}$, fourth-order elastic stiffness tensors $\mathbb{C}^{i}$, and volume $V_{i} . U_{c}$ is the elastic energy stored in the elastic control cable, which has the axial stiffness $k_{c}$ and is elongated by $\Delta L_{c}$, and $W_{c}$ is the work potential of the control cable, which can be readily computed as the dot product between the force vector $\mathbf{F}_{c}=\left[\begin{array}{lll}T & 0 & 0\end{array}\right]^{\mathrm{T}}$ and displacement vector $\boldsymbol{\Delta}_{c}=\left[\begin{array}{lll}\Delta d & 0 & 0\end{array}\right]^{\mathrm{T}}$.

In order to facilitate the model description, we set a stationary reference frame $\mathbf{X}_{1}-\mathbf{X}_{2}-\mathbf{X}_{3}$, whose origin sits on the center point of the composite's top surface, and the $\mathbf{X}_{1^{-}}, \mathbf{X}_{2^{-}}$, and $\mathbf{X}_{3}$-axes are oriented along with the composite's length, width, and thickness directions [Fig. 1(a)]. We also set a local reference frame $\mathbf{x}_{1}-\mathbf{x}_{2}-\mathbf{X}_{3}$, which coincides with the stationary global $\mathbf{X}_{1}-\mathbf{X}_{2}-\mathbf{X}_{3}$ reference frame in the undeformed state [Fig. 1(a)] but moves following the deformation of the composite [Fig. 1(d)]. In addition, we set a fiber reference frame $\mathbf{m}_{1}-\mathbf{m}_{2}-\mathbf{m}_{3}$ [Fig. 1(b)], where the $\mathbf{m}_{1}$-axis rotates from the $\mathbf{x}_{1}$-axis by the fiber orientation angle $\phi_{0}$ around the $\mathbf{m}_{3}$-axis. In a helically bent composite, we can always find a principle strain plane $\left[\mathbf{r}_{1}-\mathbf{r}_{2}\right.$ plane, inset of Fig. 1(d)] where the $\mathbf{r}_{3}$-axis coincides with the $\mathbf{x}_{3}$-axis and the $\mathbf{r}_{1}$-axis rotates counter-clockwise from the $\mathbf{x}_{1}$-axis by $\phi$. In this $\mathbf{r}_{1}-\mathbf{r}_{2}$ principle strain plane, there is no shear deformation. Therefore, the strain tensor only has three principle components $e_{11}$, $e_{22}$, and $e_{33}$. In helical bending, a composite bends about the $\mathbf{r}_{1}$-axis and $\mathbf{r}_{2}$-axis with curvatures $\kappa_{1}$ and $\kappa_{2}$, respectively. Based on Euler-Bernoulli beam theory, we can calculate the strain tensors in any parallel plane which offsets $x_{3}$ from the $\mathbf{r}_{1}-\mathbf{r}_{2}$ principle strain plane as

$$
\boldsymbol{\varepsilon}^{(r)}=\varepsilon_{11}^{(r)} \mathbf{r}_{1} \otimes \mathbf{r}_{1}+\varepsilon_{22}^{(r)} \mathbf{r}_{2} \otimes \mathbf{r}_{2}+\varepsilon_{33}^{(r)} \mathbf{r}_{3} \otimes \mathbf{r}_{3},
$$

where $\varepsilon_{11}^{(r)}\left(x_{3}\right)=e_{11}+x_{3} \kappa_{1}, \varepsilon_{22}^{(r)}\left(x_{3}\right)=e_{22}+x_{3} \kappa_{2}$, and $\varepsilon_{33}^{(r)}\left(x_{3}\right)$ $=e_{33}+x_{3} q$. Here, $q$ denotes the gradient of the strain component along the $\mathbf{x}_{3}$-axis, which is required for plane strain compatibility. Using $\varepsilon_{11}^{(r)}, \varepsilon_{22}^{(r)}$, and $\varepsilon_{33}^{(r)}$, we can calculate the strain components $\varepsilon_{i j}$ of the strain tensor $\boldsymbol{\varepsilon}\left(\boldsymbol{\varepsilon}=\varepsilon_{i j} \mathbf{x}_{i} \otimes \mathbf{x}_{j}\right)$ in the $\mathbf{x}_{1}-\mathbf{x}_{2}-\mathbf{x}_{3}$ frame through the tensor coordinate transformation,

$$
\varepsilon_{i j}=Q_{m i}^{(1)} Q_{n j}^{(1)} \varepsilon_{m n}^{(r)} .
$$

Here, $Q_{i j}^{(1)}$ are the components of the orthogonal tensor $\mathbf{Q}^{(1)}$ that can be used to rotate the vectors in the $\mathbf{r}_{1}-\mathbf{r}_{2}-\mathbf{r}_{3}$ frame to the $\mathbf{x}_{1}-\mathbf{x}_{2}-\mathbf{x}_{3}$ frame by an angle $\phi . \mathbf{Q}^{(1)}$ includes only the non-zero components $Q_{11}^{(1)}=Q_{22}^{(1)}=\cos \phi, \quad Q_{12}^{(1)}=-Q_{12}^{(1)}$ $=\sin \phi$, and $Q_{33}^{(1)}=1$.

As the top and bottom layers are made of an isotropic elastic matrix material, the fourth-order stiffness tensor $\mathbb{C}^{i}$ $(i=1,3)$ can be readily calculated using the Young's modulus $E_{m}$ and Poisson ratio $\nu_{m}$ as shown in matrix form in the supplementary material (S4). Thus, the elastic energy $U_{i}$ $(i=1,3)$ stored in the top and bottom layers can be computed based on Eq. (1)

$$
\begin{aligned}
U_{i}= & \int\left\{\frac{1}{2}\left(\lambda_{m}+2 \mu_{m}\right)\left[\left(\varepsilon_{11}^{(r)}\right)^{2}+\left(\varepsilon_{22}^{(r)}\right)^{2}+\left(\varepsilon_{33}^{(r)}\right)^{2}\right]\right. \\
& \left.+\lambda_{m}\left(\varepsilon_{11}^{(r)} \varepsilon_{22}^{(r)}+\varepsilon_{22}^{(r)} \varepsilon_{33}^{(r)}+\varepsilon_{33}^{(r)} \varepsilon_{11}^{(r)}\right)\right\} d V_{i}
\end{aligned}
$$

where $\lambda_{m}=E_{m} \nu_{m} /\left(1+\nu_{m}\right)\left(1-2 \nu_{m}\right)$ and $\mu_{m}=E_{m} / 2\left(1+\nu_{m}\right)$. The middle layer is a fiber reinforced orthotropic composite which is designed by rotating the basic fiber reinforced composite by $\phi_{0}$ in the counter-clockwise direction [Figs. 1(b) and 1(c)]. The fourth-order stiffness tensor $\mathbb{C}^{2}$ of the basic composite can be expressed in a Voigt matrix notation with nine independent parameters $C_{11}, C_{12}, C_{13}, C_{22}, C_{23}, C_{33}$, $C_{44}, C_{55}$, and $C_{66}$, which can be readily calculated using the experimentally measured parameters (see supplementary material, S5). In addition, using the coordinate transformation tensor $\mathbf{Q}^{(2)}$, which includes only the non-zero components $Q_{11}^{(2)}=Q_{22}^{(2)}=\cos \left(\phi_{0}-\phi\right), Q_{12}^{(2)}=-Q_{12}^{(2)}=-\sin \left(\phi_{0}-\phi\right)$, $Q_{33}^{(2)}=1$, we can express the strain tensor $\boldsymbol{\varepsilon}^{(m)}\left(\boldsymbol{\varepsilon}^{(m)}=\varepsilon_{i j}^{(m)}\right.$ $\left.\mathbf{m}_{i} \otimes \mathbf{m}_{j}\right)$ in the $\mathbf{m}_{1}-\mathbf{m}_{2}-\mathbf{m}_{3}$ frame in terms of the strain tensor $\boldsymbol{\varepsilon}^{(r)}$ in the $\mathbf{r}_{1}-\mathbf{r}_{2}-\mathbf{r}_{3}$ frame

$$
\varepsilon_{i j}^{(m)}=Q_{m i}^{(2)} Q_{n j}^{(2)} \gamma_{m n}^{(r)} .
$$

The elastic energy $U_{2}$ stored in the middle layer described as $\int \frac{1}{2} \varepsilon^{2}: \mathbb{C}^{2}: \boldsymbol{\varepsilon}^{2} d V_{2}$ in Eq. (1) can also be expressed as $\int \frac{1}{2} \boldsymbol{\varepsilon}^{2(m)}: \mathbb{C}^{2}: \boldsymbol{\varepsilon}^{2(m)} d V_{2}$, as it is independent of the reference frames. The complete expression of $U_{2}$ is shown in the supplementary material (S4).

At the position $x_{3}=-h_{c}$, the application of the external force $T$ to the control cable results in the helical motion of a composite actuator. The elongation $\Delta L_{c}$ of the control cable is $T / k_{c}$, where the axial stiffness $k_{c}$ was measured from an experiment (see supplementary material, S5). Therefore, the elastic energy $U_{c}$ stored in the deformed cable is $T^{2} /\left(2 k_{c}\right)$. Moreover, based on Eqs. (2) and (3), we can calculate the normal strain component along the $\mathbf{x}_{1}$-axis $\varepsilon_{11}$ at $x_{3}=-h_{c}$ as $\varepsilon_{11}\left(x_{3}=-h_{c}\right)=\varepsilon_{11}^{(r)}\left(x_{3}=-h_{c}\right) \cos ^{2} \phi+\varepsilon_{22}^{(r)}\left(x_{3}=-h_{c}\right) \sin ^{2} \phi$. The displacement $\Delta d$ of the external force $T$ acting on the cable is $\varepsilon_{11}\left(x_{3}=-h_{c}\right) \cdot L+\Delta L_{c}$. The work potential $W_{c}$ by the force $T$ is

$$
W_{c}=L T\left[\left(e_{11}-h_{c} \kappa_{1}\right) \cos ^{2} \phi+\left(e_{22}-h_{c} \kappa_{2}\right) \sin ^{2} \phi\right]+T^{2} / k_{c} .
$$


In summary, the total potential energy of a composite actuator is a function of seven unknowns, i.e., $\Pi=\Pi\left(\kappa_{1}\right.$, $\left.\kappa_{2}, q, e_{11}, e_{22}, e_{33}, \phi\right)$. The composite actuator arrives at the equilibrium when the total potential energy is minimized, which means

$$
\begin{gathered}
\frac{\partial \Pi}{\partial \kappa_{1}}=0, \quad \frac{\partial \Pi}{\partial \kappa_{2}}=0, \quad \frac{\partial \Pi}{\partial q}=0, \quad \frac{\partial \Pi}{\partial e_{11}}=0, \\
\frac{\partial \Pi}{\partial e_{22}}=0, \quad \frac{\partial \Pi}{\partial e_{33}}=0, \quad \frac{\partial \Pi}{\partial \phi}=0 .
\end{gathered}
$$

By solving the seven equations in Eq. (7) numerically, we can obtain the seven unknowns to express the total potential energy. Using $\kappa_{1}, \kappa_{2}$, and $\phi$, we can calculate the magnitude of pitch $P$ and radius $R$ of a helical deformation (supplementary material, S7)

$$
\begin{gathered}
|P|=2 \pi \frac{\left(\kappa_{1}-\kappa_{2}\right) \sin 2 \phi}{\kappa_{1}^{2}+\kappa_{2}^{2}+\left(\kappa_{1}-\kappa_{2}\right)\left(\kappa_{1}+\kappa_{2}\right) \cos 2 \phi}, \\
R=\frac{\kappa_{1}+\kappa_{2}+\left(\kappa_{1}-\kappa_{2}\right) \cos 2 \phi}{\kappa_{1}^{2}+\kappa_{2}^{2}+\left(\kappa_{1}-\kappa_{2}\right)\left(\kappa_{1}+\kappa_{2}\right) \cos 2 \phi} .
\end{gathered}
$$

Here, $P$ is positive if the actuator twist towards the positive $\mathbf{X}_{2}$-direction. The calculated $P$ and $R$ can be further used to compute the slope $S$ and the curvature $\kappa$.

With the material parameters measured from experiments (see supplementary material, S5), our theoretical framework can predict the helical deformations with different fiber orientation angles $\phi_{0}$ and different control cable displacements $\Delta d$, respectively. In Figs. 2(a) and 2(b), the model predictions qualitatively agree with the experimental results. We also constructed the deformed 3D geometries (see supplementary materials, S6). In Figs. 2(c) and 2(d), the model predictions demonstrate good agreement with experimental snapshots.

The validated theoretical framework can be further used as a design tool to guide the design of helical deformations of cable-driven anisotropic composite actuators. The effects of geometrical/material parameters fiber volume fraction $v_{f}$, fiber stiffness $E_{f}$, cable position $h_{c}$, and middle layer position $h_{2}$ on the helical behavior are studied. In all the parametric studies, we keep the external force constant $(F=16 \mathrm{~N})$ and assume that the stiffness of the control fiber is infinite. The effect of the control cable position $h_{c}$ and the middle layer position $h_{2}$ on the helical deformation is only shown in the supplementary material (S7).

In Fig. 3, we explore the effects of the middle layer's fiber volume fraction $v_{f}$. The insets of Figs. 3(a) and 3(b) present the overview of the effect of $v_{f}$ on the slope $S$ and the curvature $\kappa$ of the composites with different orientation angles $\phi_{0}$. Figure 3(a) suggests three key insights into the effect of $v_{f}$ on the slope $S$ : (i) the composite exhibits in-plane bending not only at $\phi_{0}=0^{\circ}$ and $90^{\circ}$ but also at $\phi_{0}=\sim 55^{\circ}$. The previous study explains this angle as a turning point, where the fibers only perform rotational deformation rather than elongation; ${ }^{40}$ (ii) between $\phi_{0}=0^{\circ}$ and $55^{\circ}$, the composite actuator deforms helically with a positive upward slope, and between $\phi_{0}=55^{\circ}$ and $90^{\circ}$, the helical deformation is
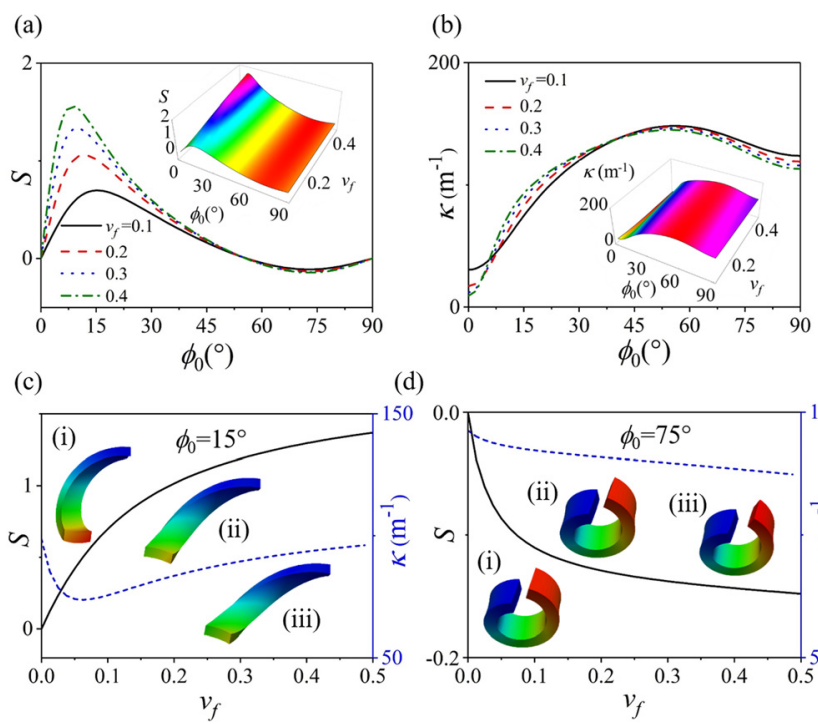

(d)

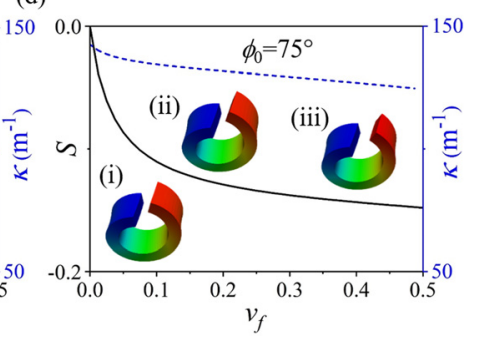

FIG. 3. The dependence of (a) slope $S$ and (b) curvature $\kappa$ on fiber orientation angle $\phi_{0}$ for different fiber volume fractions $v_{f}=0.1,0.2,0.3$, and 0.4 ; the insets show the corresponding 3D figures. The dependence of $S$ and $\kappa$ on $v_{f}$ for soft actuators with (c) $\phi_{0}=15^{\circ}$ and (d) $75^{\circ}$ is shown; the insets show deformed shapes with $v_{f}=0.1$ (i), 0.3 (ii), and 0.5 (iii).

opposite; (iii) the increase in $v_{f}$ leads to a remarkable increase in the positive upward slope $S$ between $\phi_{0}=0^{\circ}$ and $55^{\circ}$, while the increase in the negative downward slope $S$ between $\phi_{0}=55^{\circ}$ and $90^{\circ}$ is only small. In Fig. 3(b), the increase in $v_{f}$ does not cause a significant increase in curvature $\kappa$, and the maximum $\kappa$ always occurs at $\phi_{0}=55^{\circ}$. Figures 3(c) and 3(d) demonstrate the effect of $v_{f}$ on $S$ and $\kappa$ for the two particular composites with $\phi_{0}=15^{\circ}$ and $75^{\circ}$. In Fig. 3(c), the increase in $v_{f}$ leads to a continuous increase in slope $S$ when $\phi_{0}=15^{\circ}$, while the curvature $\kappa$ first decreases to a minimum at $v_{f}=0.06$ and then increases with the increase in $v_{f}$. In contrast, the change in $S$ and $\kappa$ is relatively small at $\phi_{0}=75^{\circ}$, and the increase in $v_{f}$ results in a continuous decrease in $\kappa$. In the insets of Figs. 3(c) and 3(d), the deformed 3D representations clearly illustrate the effect of $v_{f}$ on the helical deformations of the two composites.

Figures 4(a) and 4(b) present the effect of the fiber stiffness $E_{f}$ on the slope $S$ and the curvature $\kappa$, with $E_{f}$ varying from $1 / 100 E_{f 0}$ to $10 E_{f 0}$, where $E_{f 0}$ is the original fiber stiffness $\left(E_{f 0}=1.2 \mathrm{GPa}\right)$. In general, the increase in $E_{f}$ results in greater anisotropy, thus significantly increasing $S$. This trend is reflected by the deformed 3D representations, whose orientation angles are $15^{\circ}$ [Fig. 4(c)] and $75^{\circ}$ [Fig. 4(d)]. In Fig. 4(c), it is also found that at $\phi_{0}=15^{\circ}$, the minimum $\kappa$ exists at a moderate $E_{f}=10^{-0.75} E_{f 0}$. This is due to the competition between the bending modulus, which is increased with the increase in $E_{f}$, and the bending moment, which is also increased as the larger $E_{f}$ shifts the neutral plane away from the external force application point.

In summary, we developed a theoretical framework based on the minimum potential energy method to model the controllable helical deformation of cable-driven anisotropic composite actuators. We validated the framework with experimental results for multimaterial 3D printed composites, captured from a self-built experimental platform. The framework reproduces helical deformations with different 
(a)

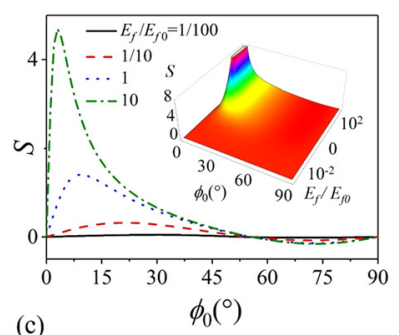

(c)

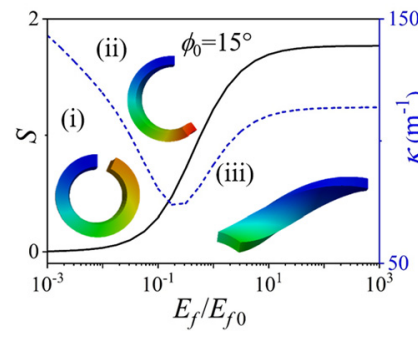

(b)

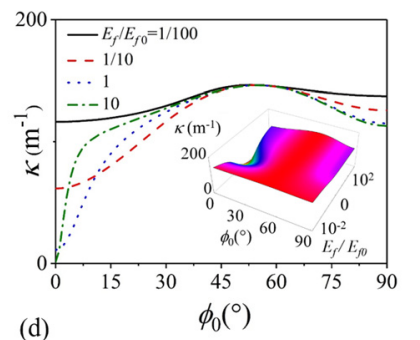

(d)

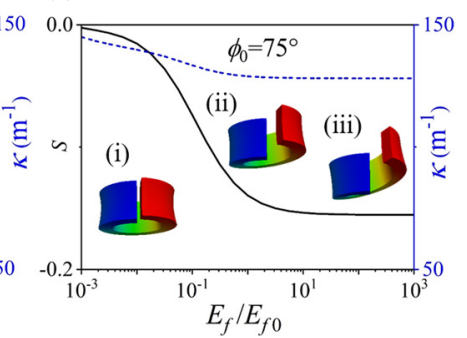

FIG. 4. The dependence of (e) $S$ and (f) $\kappa$ on $\phi_{0}$ for different fiber elastic moduli $E_{f}=1 / 100 E_{f 0}, 1 / 10 E_{f 0}, E_{f 0}$, and $10 E_{f 0}$; the insets show the corresponding 3D figures. The dependence of $S$ and $\kappa$ on $E_{f} E_{f 0}$ for soft actuators with (g) $\phi_{0}=15^{\circ}$ and (h) $75^{\circ}$ is shown; the insets show deformed shapes with $E_{f} / E_{f 0}=10^{-2}$ (i), $10^{-1}$ (ii), and $10^{3}$ (iii).

design parameters. We further use the developed framework to investigate the effects of material and geometric parameters on helical deformations.

See supplementary material for additional information on analytical modeling and experiments.

D.W., A.S., O.W., and Q.G. acknowledge the support from the SUTD Digital Manufacturing and Design Centre (DManD), supported by the Singapore National Research Foundation. Q.G. acknowledges the Startup Research Grant from the Singapore University of Technology and Design, the Research Project of State Key Laboratory of Mechanical System, and Vibration MSV201802 by Shanghai Jiao Tong University and the research fund from the U.S. Office of Naval Research Global (ONRG). G.Y.G. acknowledges research grants from the National Natural Science Foundation of China (Grant No. 51622506) and the Science and Technology Commission of Shanghai Municipality (Grant No. 16JC1401000).

${ }^{1}$ J. D. Watson and F. H. Crick, Nature 171, 737 (1953).

${ }^{2}$ N. L. Nerurkar, L. Mahadevan, and C. J. Tabin, Proc. Natl. Acad. Sci. 114, 2277 (2017).

${ }^{3}$ T. Savin, N. A. Kurpios, A. E. Shyer, P. Florescu, H. Liang, L. Mahadevan, and C. J. Tabin, Nature 476, 57 (2011).

${ }^{4}$ S. Armon, E. Efrati, R. Kupferman, and E. Sharon, Science 333, 1726 (2011).

${ }^{5}$ W. Jung, S. M. Choi, W. Kim, and H.-Y. Kim, Phys. Fluids 29, 041702 (2017).

${ }^{6}$ N. Chouaieb, A. Goriely, and J. H. Maddocks, Proc. Natl. Acad. Sci. 103, 9398 (2006).

${ }^{7}$ T. Cook, The Curves of Life, Constable, London, 1914, Reprinted (Dover, London, 1979).

${ }^{8}$ Y. Forterre and J. Dumais, Science 333, 1715 (2011).

${ }^{9}$ P. Chen, Y. Xu, S. He, X. Sun, S. Pan, J. Deng, D. Chen, and H. Peng, Nat. Nanotechnol. 10, 1077 (2015).

${ }^{10}$ C. Schmuck, Angew. Chem. Int. Ed. 42, 2448 (2003).

${ }^{11}$ R. Oda, I. Huc, M. Schmutz, S. Candau, and F. MacKintosh, Nature 399, 566 (1999).

${ }^{12}$ N. H. Mendelson, Sci. Prog. 74, 425 (1990).

${ }^{13}$ M. Tabor and I. Klapper, IMA Vol. Math. Appl. 82, 139 (1996).

${ }^{14}$ N. H. Mendelson, Proc. Natl. Acad. Sci. 75, 2478 (1978).

${ }^{15}$ M. W. Hannan and I. D. Walker, J. Field Rob. 20, 45 (2003).

${ }^{16}$ J. L. Silverberg, R. D. Noar, M. S. Packer, M. J. Harrison, C. L. Henley, I. Cohen, and S. J. Gerbode, Proc. Natl. Acad. Sci. 109, 16794 (2012).

${ }^{17}$ J. P. Mesirov, K. Schulten, and D. W. Sumners, Mathematical Approaches to Biomolecular Structure and Dynamics (Springer Science \& Business Media, 2012), Vol. 82.

${ }^{18}$ Y. Abraham, C. Tamburu, E. Klein, J. W. C. Dunlop, P. Fratzl, U. Raviv, and R. Elbaum, J. R. Soc. Interface 9, 640 (2012).

${ }^{19}$ K. C. Galloway, K. P. Becker, B. Phillips, J. Kirby, S. Licht, D. Tchernov, R. J. Wood, and D. F. Gruber, Soft Rob. 3, 23 (2016).

${ }^{20}$ H. Yuk, S. Lin, C. Ma, M. Takaffoli, N. X. Fang, and X. Zhao, Nat. Commun. 8, 14230 (2017).

${ }^{21}$ B. Gorissen, T. Chishiro, S. Shimomura, D. Reynaerts, M. De Volder, and S. Konishi, Sens. Actuators A: Phys. 216, 426 (2014).

${ }^{22}$ V. Palmre, J. J. Hubbard, M. Fleming, D. Pugal, S. Kim, K. J. Kim, and K. K. Leang, Smart Mater. Struct. 22, 014003 (2013).

${ }^{23}$ P. Polygerinos, Z. Wang, K. C. Galloway, R. J. Wood, and C. J. Walsh, Rob. Auton. Syst. 73, 135 (2015).

${ }^{24}$ J. S. Wang, X. Q. Feng, G. F. Wang, and S. W. Yu, Appl. Phys. Lett. 92, 191901 (2008).

${ }^{25}$ Z. Chen, Q. Guo, C. Majidi, W. Chen, D. J. Srolovitz, and M. P. Haataja, Phys. Rev. Lett. 109, 114302 (2012).

${ }^{26}$ X. Y. Ji, M. Q. Zhao, F. Wei, and X. Q. Feng, Appl. Phys. Lett. 100, 263104 (2012).

${ }^{27}$ S. Kim, W. Kim, H. Chung, and M. Cho, RSC Adv. 5, 96387 (2015).

${ }^{28}$ S. Iamsaard, E. Villemin, F. Lancia, S. Asshoff, S. P. Fletcher, and N. Katsonis, Nat. Protoc. 11, 1788 (2016).

${ }^{29}$ L. Liu, A. Ghaemi, S. Gekle, and S. Agarwal, Adv. Mater. 28, 9792 (2016).

${ }^{30}$ L. Zhang, S. Chizhik, Y. Wen, and P. Naumov, Adv. Funct. Mater. 26, 1040 (2016).

${ }^{31}$ Q. Ge, H. J. Qi, and M. L. Dunn, Appl. Phys. Lett. 103, 131901 (2013).

${ }^{32}$ J.-S. Wang, H.-M. Ye, Q.-H. Qin, J. Xu, and X.-Q. Feng, Proc. R. Soc. A: Math., Phys. Eng. Sci. 468, 609 (2012).

${ }^{33}$ D. B. Camarillo, C. F. Milne, C. R. Carlson, M. R. Zinn, and J. K. Salisbury, IEEE Trans. Rob. 24, 1262 (2008).

${ }^{34}$ C. Cipriani, M. Controzzi, and M. C. Carrozza, Robotica 28, 919 (2010).

${ }^{35}$ A. S. Boxerbaum, K. M. Shaw, H. J. Chiel, and R. D. Quinn, Int. J. Rob. Res. 31, 302 (2012).

${ }^{36}$ G. Palli, G. Borghesan, and C. Melchiorri, IEEE Trans. Rob. 28, 277 (2012).

${ }^{37}$ M. Cianchetti, M. Calisti, L. Margheri, M. Kuba, and C. Laschi, Bioinspiration Biomimetics 10, 035003 (2015).

${ }^{38}$ H. In, B. B. Kang, M. Sin, and K.-J. Cho, IEEE Rob. Autom. Mag. 22, 97 (2015).

${ }^{39}$ C. Laschi, B. Mazzolai, and M. Cianchetti, Sci. Rob. 1, eaah3690 (2016).

${ }^{40}$ Q. Ge, A. Serjouei, H. J. Qi, and M. L. Dunn, Int. J. Solids Struct. 102-103, 186 (2016).

${ }^{41}$ Q. Ge, C. K. Dunn, H. J. Qi, and M. L. Dunn, Smart Mater. Struct. 23, 094007 (2014).

${ }^{42}$ J. N. Reddy, An Introduction to Continuum Mechanics (Cambridge University Press, 2013).

${ }^{43}$ J. N. Reddy, Energy Principles and Variational Methods in Applied Mechanics (John Wiley \& Sons, 2017).

This article may be downloaded for personal use only. Any other use requires prior permission of the author and AIP Publishing. This article appeared in Appl. Phys. Lett. 112, 181905 (2018) and may be found at doi: 10.1063/1.5025370. 